\title{
Quantitative Pooling of Michaelis-Menten Equations in Models with Parallel Metabolite Formation Paths
}

\author{
Allen J. Sedman' ${ }^{1}$ and John G. Wagner ${ }^{1,2}$ \\ Received Sept. 4, 1973-Final Jan. 11, 1974
}

\begin{abstract}
Pooling of Michaelis-Menten equations for models having parallel paths for formation of two or more metabolites is discussed. A theory which explains phenomena exhibited by pooled nonlinear pharmacokinetic systems and equations relating pooled Michaelis-Menten constants $\left(V_{p}, K_{p}\right)$ to microscopic constants $\left(V_{i}, K_{i}\right)$ are presented. The suitability of this type of pooling for use in pharmacokinetic modeling is also discussed. Use of pooling concepts in the design of clinical studies is demonstrated.
\end{abstract}

KEY WORDS: Michaelis-Menten kinetics; dose-dependent kinetics; pooling of nonlinear equations.

\section{INTRODUCTION}

Previous authors (1-6) have shown that accurate fitting of some plasma concentration-time $(C, t)$ data observed following administration of two or more doses of drug involves nonlinear Michaelis-Menten elimination. The application of first-order kinetics to such data can result in one type of "dose-dependent" kinetics $(4,6)$. Although nonlinear pharmacokinetics allows $C, t$ data to be accurately described, the analysis of data becomes much more complex than in classical linear pharmacokinetics. In the nonlinear case, differential equations can often be utilized only by means of numerical integration techniques and, preferably, a digital computer. Computer time required for fitting is usually much longer than with linear equations. In addition, as shown for salicylate by Levy et al. $(2,3)$, the large amount of laboratory time needed to define a nonlinear model becomes almost prohibitive.

Supported in part by Public Health Service Grant 5-P11-GM15559.

${ }^{1}$ College of Pharmacy and Upjohn Center for Clinical Pharmacology, The University of Michigan, Ann Arbor, Michigan 48104.

${ }^{2}$ Address requests for reprints to Dr. John G. Wagner, Upjohn Center for Clinical Pharmacology, The University of Michigan, Ann Arbor, Michigan 48104. 
In classical first-order models, simplification is often achieved by the addition of first-order rate constants applicable to parallel paths for formation of two or more metabolites from the drug. Simplification of parallel nonlinear systems in a similar manner would obviously be desirable. However, because Michaelis-Menten equations are nonlinear, one would not expect the constants to be additive. Methods for obtaining kinetic constants for two or more enzymes acting on the same substrate are discussed in the literature (7-10). In general, these reports are concerned with isolated (in vitro) systems which provide Lineweaver-Burk plots characterized by marked nonlinearities. Iterative computer programs are used to resolve nonlinear Lineweaver-Burk plots. Application of these techniques to estimate kinetic constants for metabolite formation involving two or more enzymes using in vivo $C, t$ data would be difficult for the following reasons: First, resolution of the segments of nonlinear Lineweaver-Burk plots becomes very difficult when the Michaelis constant for one enzyme approaches that for another enzyme and/or when the maximum velocities approach each other (7). Resolution would be extremely difficult when several enzymes acted on the same substrate. Second, in vivo data are generally more scattered than in vitro data. Therefore, it might be difficult to determine whether a Lineweaver-Burk plot is linear or nonlinear with appropriate statistical methods. Moreover, the estimation of enzyme constants from scattered Lineweaver-Burk data would be difficult, and the standard deviations of the constants could exceed the estimates by severalfold. Third, the use of Lineweaver-Burk techniques is inappropriate for drugs described by multicompartment models (11).

Pharmacokinetic modeling does not always require the elucidation of a complete model. In general, the simplest model that accurately describes the $C, t$ data obtained following administration of one or more doses of drug, and which has predictive properties, is all that is needed. The purposes of this report are as follows:

1. To determine the consequences of combining or pooling MichaelisMenten equations applicable to the same substrate.

2. To develop a theory to explain the characteristics exhibited by pooled nonlinear pharmacokinetic systems.

3. To determine whether simplification of such systems can be of use in the design of clinical studies. It should be noted that an investigator is often forced to pool various metabolic paths since all the metabolites have not been isolated and identified.

\section{THEORY}

A one-compartment open model involving $n$ parallel MichaelisMenten paths is shown schematically in scheme 1: 


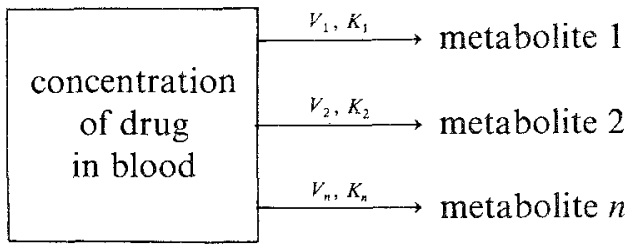

(Scheme 1)

This model is described mathematically by equation 1 :

$$
-\frac{d C}{d t}=\frac{V_{1} C}{K_{1}+C}+\frac{V_{2} C}{K_{2}+C}+\cdots+\frac{V_{n} C}{K_{n}+C}
$$

Equation 1 may also be written as equation 2:

$$
-\frac{d C}{d t}=\sum_{i=1}^{n}\left(\frac{V_{i} C}{K_{i}+C}\right)
$$

In equation 2, $n$ is the number of parallel Michaelis-Menten paths (scheme 1), $V_{i}$ is the maximum velocity of the $i$ th Michaelis-Menten equation, $K_{i}$ is the Michaelis constant for the $i$ th equation, $C$ represents the concentration of unchanged drug in the blood, and $t$ is time.

The model shown in scheme 2 results from the pooling of the parallel paths depicted in scheme 1 :

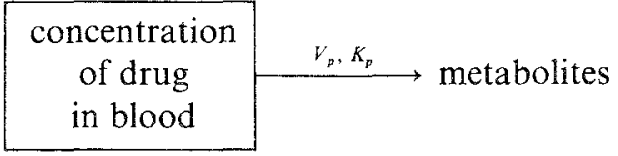

(Scheme 2)

Equation 3 mathematically describes scheme 2:

$$
-\frac{d C}{d t}=\frac{V_{p} C}{K_{p}+C}
$$

In equation $3, V_{p}$ represents the pooled maximum velocity and $K_{p}$ represents the pooled Michaelis constant. The other symbols are as defined above.

If $n$ Michaelis-Menten equations can be represented by a single pooled equation, then equation 2 must be equivalent to equation 3 . A point-forpoint correspondence is also implied. Equating the right-hand sides of equations 2 and 3 leads to equation 4 :

$$
\frac{V_{p} C}{K_{p}+C}=\sum_{i=1}^{n}\left(\frac{V_{i} C}{K_{i}+C}\right)
$$

Division of both sides of equation 4 by $C$ provides equation 5:

$$
\frac{V_{p}}{K_{p}+C}=\sum_{i=1}^{n}\left(\frac{V_{i}}{K_{i}+C}\right)
$$


Imposing boundary conditions in equation 5 leads to equations 6 and 7 . When $t=0, C=C_{0}$, and substitution into equation 5 yields

$$
\frac{V_{p}}{K_{p}+C_{0}}=\sum_{i=1}^{n}\left(\frac{V_{i}}{K_{i}+C_{0}}\right)
$$

When $t=\infty, C=0$, and substitution into equation 5 yields

$$
\frac{V_{p}}{K_{p}}=\sum_{i=1}^{n}\left(\frac{V_{i}}{K_{i}}\right)
$$

Solving equations 6 and 7 for $V_{p}$ and $K_{p}$, respectively, gives the dosedependent equations 8 and 9 :

$$
\begin{aligned}
& V_{p}=C_{0}\left[\sum_{i=1}^{n}\left(\frac{V_{i}}{K_{i}+C_{0}}\right)\right]\left[\sum_{i=1}^{n}\left(\frac{V_{i}}{K_{i}}\right)\right] /\left[\sum_{i=1}^{n}\left(\frac{V_{i}}{K_{i}}\right)-\sum_{i=1}^{n}\left(\frac{V_{i}}{K_{i}+C_{0}}\right)\right] \\
& K_{p}=C_{0}\left[\sum_{i=1}^{n}\left(\frac{V_{i}}{K_{i}+C_{0}}\right)\right] /\left[\sum_{i=1}^{n}\left(\frac{V_{i}}{K_{i}}\right)-\sum_{i=1}^{n}\left(\frac{V_{i}}{K_{i}+C_{0}}\right)\right]
\end{aligned}
$$

In equations 8 and $9, V_{p}$ and $K_{p}$ are dependent on the initial drug concentration, $C_{0}$, and hence also on the dose of the drug.

However, when $C_{0}$ is very much greater than $K_{p}$ and all values of $K_{i}$, then equations 8 and 9 simplify to the dose-independent equations 10 and 11 , respectively:

$$
\begin{gathered}
V_{p}=\sum_{i=1}^{n}\left(V_{i}\right) \\
K_{p}=\sum_{i=1}^{n}\left(V_{i}\right) / \sum_{i=1}^{n}\left(\frac{V_{i}}{K_{i}}\right)
\end{gathered}
$$

Equations 10 and 11 relate the pooled constants, $V_{p}$ and $K_{p}$, only to the microscopic constants, $V_{i}$ and $K_{i}$. In this case, $V_{p}$ and $K_{p}$ are independent of $C_{0}$.

Therefore, if nonlinear pharmacokinetic systems involving parallel Michaelis-Menten metabolism paths can be simplified in a manner similar to that employed for linear systems, equations 8 and 9 (and sometimes equations 10 and 11) should describe the relationship between the pooled parameters, $V_{p}$ and $K_{p}$, and the microscopic constants, $V_{i}$ and $K_{i}$. 


\section{EXPERIMENTAL}

\section{Generation of Simulated $C, t$ Data}

When $n=2$ in scheme 1 , the reduced model shown in scheme 3 is obtained:

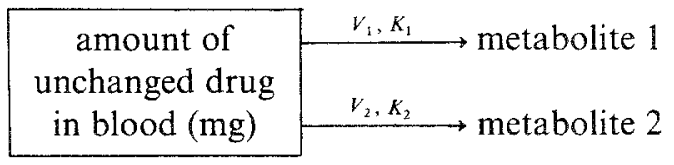

(Scheme 3)

The equation applicable to scheme 3 is given as equation 12:

$$
-\frac{d C}{d t}=\frac{V_{1} C}{K_{1}+C}+\frac{V_{2} C}{K_{2}+C}
$$

Over 100 simulations were performed by assigning numerical values to $V_{1}, V_{2}, K_{1}, K_{2}$, and $C_{0}$ in equation 12 and generating sets of $C, t$ data by numerical integration of equation 12 using the program NONLIN and an IBM 360/67 digital computer. Some of the values employed were $V_{1}=5$; $K_{1}=5 ; V_{2}=0.1,5$, and $250 ; K_{2}=5,10,15,25,50$, and $250 ;$ and $C_{0}=5$ and 250 .

\section{Fitting of Simulated $C, t$ Data}

Integration of equation 3 gives equation 13 :

$$
C_{0}-C+K_{p} \ln \left(C_{0} / C\right)=V_{p} t
$$

Each set of data, simulated as discussed above, was fitted to equation 13 by numerical integration of equation 3 using the program NONLIN and an IBM 360/67 digital computer. Initial estimates of $V_{p}$ and $K_{p}$ were obtained from equations 10 and 11 using the appropriate values of $V_{i}$ and $K_{i}$ employed in each particular simulation.

\section{Representative Example}

The parameter values (scheme 3 and equation 12) used in this representative example were $V_{1}=5, K_{1}=5, V_{2}=5, K_{2}=15$, and $C_{0}=5$.

\section{Dose-Independent Example}

Values of constants reported by Levy et al. (3) for formation of salicylurate and phenolic glucuronide from salicylate, namely $V_{1}=68, K_{1}=340$, $V_{2}=31$, and $K_{2}=542$, were used to generate simulated $A, t$ data by numerical integration of equation 12. Initial conditions ( $A_{0}$ values) employed 
were $5,50,100,300,1000$, and $\infty$ when equations 8 and 9 were applied. In this case, $A_{0}$ represents the initial amount of drug in the body and $A$ represents the amount in the body at time $t$. The $A, t$ data generated when $A_{0}=5,100$, and 1000 were fitted to equation 13 by numerical integration of equation 3 using the program NONLIN and an IBM 360/67 digital computer.

\section{Dose-Dependent Example}

Values of $V_{1}=5, K_{1}=5, V_{2}=50$, and $K_{2}=50$ were used to generate simulated $A, t$ data by numerical integration of equation 12. Initial conditions employed were $5,10,50,100,250,500$, and $\infty$ when equations 8 and 9 were applied. The $A, t$ data generated when $A_{0}=5$ and 250 were fitted to equation 13 as described above.

\section{RESULTS AND DISCUSSION}

A representative set of simulated data is plotted in Fig. 1. Cartesian coordinate plots of all sets of data simulated from equations describing parallel metabolite formation paths were characterized by the "hockeystick" shape illustrated in Fig. 1. Wagner (6) has shown that this "hockeystick" shape is characteristic of $C, t$ data generated by numerical integration of the Michaelis-Menten equation itself. This suggested that data generated by numerical integration of equation 12 might also be fitted quite well to equation 13. When the data shown in Fig. 1 were fitted to equation 13 by numerical integration of equation 3 , the estimated parameters shown in Table I were obtained. A comparison of the original simulated data and the model-predicted concentrations is made in Table II. This set of data was characterized by the excellence of fit $\left(r^{2}=1.00 \text { and Corr }=1.00\right)^{3}$ and small deviations at any point. Simulated data could generally be classified as follows: (a) dose independent and (b) dose dependent. Most sets of data, particularly the dose-independent type, could be accurately fitted with a single Michaelis-Menten equation. However, irrespective of data type, large deviations between simulated and model predicted concentrations were noted (Table IV) only after several orders of magnitude of concentrationtime $(C, t)$ data had been fitted. $C, t$ data collected for pharmacokinetic analysis generally span only one or two orders of magnitude. Therefore, the above discussion does not impose severe restrictions on enzyme pooling

${ }^{3} r^{2}=\left[\sum C^{2}-\sum(\hat{C}-C)^{2}\right] / C^{2}$, where $C$ represents the original simulated concentrations and $\hat{C}$ represents the concentrations predicted by nonlinear least-squares fitting to equation 13 by numerical integration of equation 3 . Corr $=$ the correlation coefficient for the linear regression of $\hat{C}$ on $C$. 


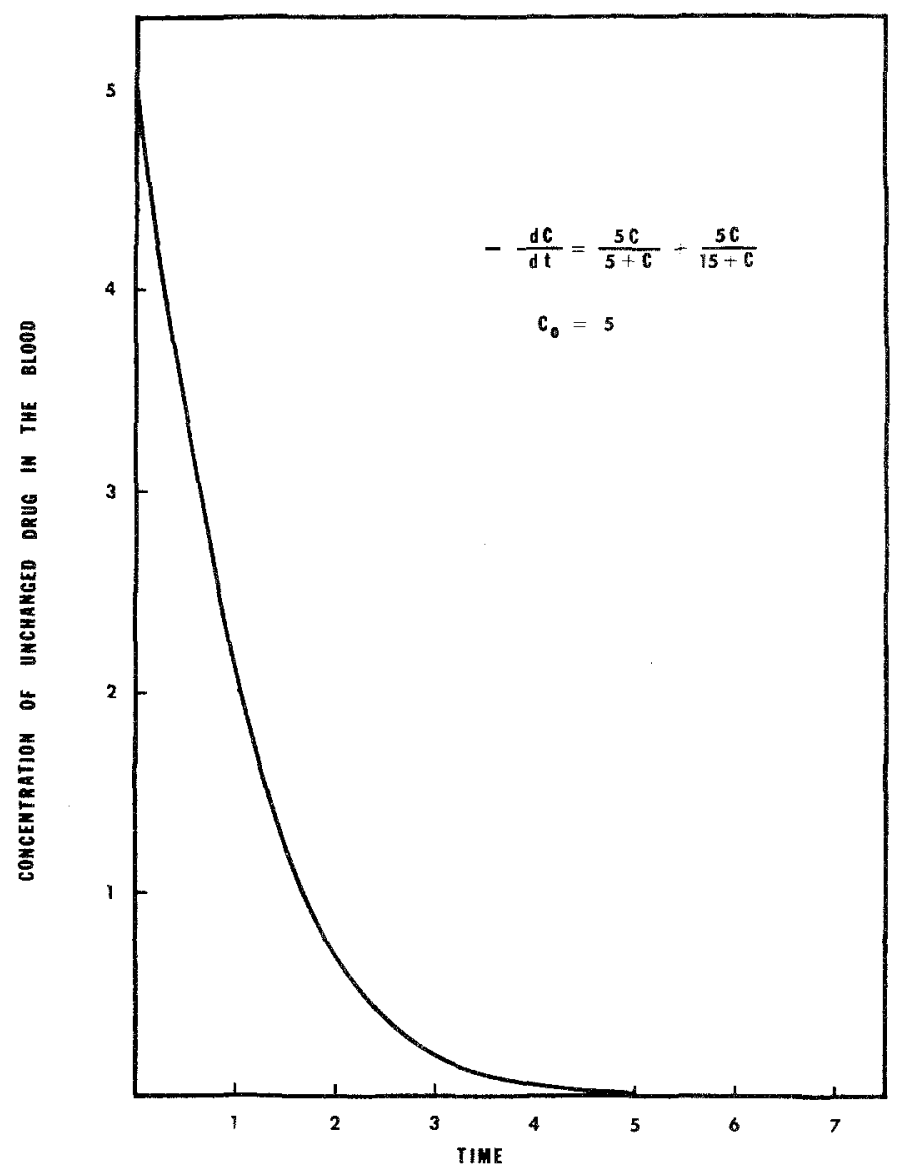

Fig. 1. Data simulated with the equation shown inset, where with reference to scheme 3 the constants are $V_{1}=5, K_{1}=5, V_{2}=5$, and $K_{2}=15$.

Table 1. Least-Squares Parameter Estimates and Their Standard Deviations Obtained for the Representative Example (see text)

\begin{tabular}{ccc}
\hline Parameter & $\begin{array}{c}\text { Estimate of } \\
\text { parameter }\end{array}$ & $\begin{array}{c}\text { Standard } \\
\text { deviation }\end{array}$ \\
\hline$C_{0}$ & 5.0020 & 0.0014 \\
$V_{p}$ & 8.5517 & 0.0030 \\
$K_{p}$ & 6.4541 & 0.0028 \\
\hline
\end{tabular}


Table II. Comparison of Simulated Drug Concentrations (generated by numerical integration of equation 12) with Fitted Values (obtained by nonlinear least-squares fitting with program NONLIN using numerical integration of equation 3$)^{a}$

\begin{tabular}{cccc}
\hline Time & $\begin{array}{c}\text { Simulated drug } \\
\text { concentration }\end{array}$ & $\begin{array}{c}\text { Fitted value for } \\
\text { drug concentration }\end{array}$ & Percent deviation \\
\hline 0 & 5.0000 & 5.0020 & -0.04 \\
0.25 & 4.1143 & 4.1184 & -0.10 \\
0.50 & 3.3339 & 3.3375 & -0.10 \\
0.75 & 2.6593 & 2.6611 & -0.06 \\
1.00 & 2.0881 & 2.0882 & 0.00 \\
1.25 & 1.6150 & 1.6137 & 0.08 \\
1.50 & 1.2316 & 1.2297 & 0.15 \\
1.75 & 0.9274 & 0.9256 & 0.19 \\
2.00 & 0.6908 & 0.6893 & 0.22 \\
2.25 & 0.5100 & 0.5090 & 0.20 \\
2.50 & 0.3737 & 0.3732 & 0.13 \\
2.75 & 0.2723 & 0.2722 & 0.04 \\
3.00 & 0.1976 & 0.1977 & 0.00 \\
3.25 & 0.1429 & 0.1432 & -0.14 \\
3.50 & 0.1030 & 0.1034 & -0.29 \\
3.75 & 0.0742 & 0.0746 & -0.40 \\
4.00 & 0.0533 & 0.0537 & -0.56 \\
4.25 & 0.0383 & 0.0387 & -0.78 \\
4.50 & 0.0275 & 0.0278 & -0.73 \\
4.75 & 0.0197 & 0.0200 & -1.02 \\
5.00 & 0.0142 & 0.0144 & -0.70 \\
\hline
\end{tabular}

$a r^{2}=1.000$, Corr $=1.000$. Data for the representative example are discussed in the text.

when it is applied to pharmacokinetic models. It will be demonstrated that enzyme pooling can yield pharmacokinetic predictions in agreement with simulated "observations." The following discussion will consider the use of enzyme pooling with respect to pharmacokinetic modeling.

The pooled parameters, $V_{p}$ and $K_{p}$, estimated by computer fitting, were always accurately predicted by equations 8 and 9 . For the representative example given, equations 8 and 9 gave $V_{p}=8.576$ and $K_{p}=6.432$, which agree very well with the computer estimates given in Table I. Simulations and fitting of simulated data were carried out only with the reduced model (scheme 3). By induction, however, it may be inferred that similar results would be obtained with simulations performed with scheme 3 and equation 1 where $n>2$. Hence the comments below should also apply to the one-compartment open model where elimination of drug is described by more than two Michaelis-Menten equations. 


\section{Dose-Independent Example}

Equations 8 and 9 indicate that $V_{p}$ and $K_{p}$ are dependent on $C_{0}$ and dose. Thus the same problem is presented as in the application of classical first-order kinetics to data described by nonlinear kinetics; i.e., the "constants" are dependent on dose. Fortunately, the time courses of data described by parallel Michaelis-Menten equations can often be closely approximated over several orders of magnitude by a single Michaelis-Menten equation with constants insensitive to large changes in dose. For this type of doseindependent data, the change in the values of the pooled constants with different doses was often equal to or less than $5 \%$. In clinical situations, because of experimental error, such small changes would not be detectable or would be unimportant. When the constants, estimated by computer fitting, changed only slightly with change in dose or $C_{0}$ value, they could be estimated by use of equations 10 and 11 .

Results of the dose-independent example are given in Table III. The following trends were noted: First, the constants changed very little with dose. As the dose increased, the magnitude of both $V_{p}$ and $K_{p}$ increased slightly. Second, the constants calculated with equations 8 and 9 agreed closely with the corresponding constants estimated by computer fitting. Third, the computer fit was excellent $\left(r^{2}=1.00\right.$ and Corr $\left.=1.00\right)$ and the maximum deviation of model-predicted amount to simulated amount was $2 \%$. Fourth, equations 10 and 11 provided values of $V_{p}=99$ and $K_{p}=385$, which are the same as those given by equations 8 and 9 when $A_{0}=\infty$. These values would be satisfactory to make predictions at any dose level.

Conditions favoring dose independence are (a) cases where the $K_{i} \mathrm{~s}$ are equal for parallel paths, (b) cases where the $K_{i}$ s are within a factor

Table III. Pooled Constants Obtained by Computer Fitting of Equation 3 and by Means of Equations 8 and $9^{a}$

\begin{tabular}{|c|c|c|c|c|c|}
\hline \multicolumn{3}{|c|}{ Computer-fitted values $^{b}$} & \multicolumn{3}{|c|}{ Calculated with equations 8 and 9} \\
\hline$A_{0}$ & $V_{p}$ & $K_{p}$ & $A_{0}$ & $V_{p}$ & $K_{p}$ \\
\hline- & - & - & $\infty$ & 99 & 384.9 \\
\hline 999.3 & 97.38 & 379.8 & 1000 & 97.78 & 380.7 \\
\hline - & - & - & 300 & 96.72 & 376 \\
\hline 100 & 95.94 & 373.1 & 100 & 95.95 & 373.1 \\
\hline - & - & - & 50 & 95.68 & 372 \\
\hline 5 & 95.38 & 370.8 & 5 & 95.37 & 370.8 \\
\hline
\end{tabular}

${ }^{a}$ Data were generated by numerical integration of equation 12 using the values of constants for salicylate given in the text (see dose-independent example in text).

${ }^{b}$ Measures of fit were $r^{2}=1.00$, Corr $=1.00 ;$ maximum deviation at any point was $2 \%$. 
of 3 of each other, and (c) systems where the drug is metabolized primarily by one enzyme.

\section{Dose-Dependent Example}

Use of a pooled model to fit data described by parallel MichaelisMenten equations with values of $K_{i}$ s which are separated by at least a factor of 5 results in dose-dependent "constants." As stated earlier and illustrated in Table IV, this type of data cannot be well represented over several orders of magnitude by a single Michaelis-Menten equation. The Lineweaver-Burk plot constructed from the concentration-time data listed in Table IV would be characterized by marked curvature. As discussed earlier, kinetic constants can easily be elucidated for systems of enzymes acting on the same substrate which provide Lineweaver-Burk plots characterized by marked nonlinearities. Therefore, since the constants for each enzyme are readily available, simplification of such systems is unnecessary.

Table IV. Comparison of Simulated Drug Concentrations (generated by numerical integration of equation 12) with Fitted Values (obtained by nonlinear least-squares fitting with program NONLIN using numerical integration of equation 3$)^{a}$

\begin{tabular}{cccc}
\hline Time & $\begin{array}{c}\text { Simulated drug } \\
\text { concentration }\end{array}$ & $\begin{array}{c}\text { Fitted values for } \\
\text { drug concentration }\end{array}$ & \multicolumn{1}{c}{ Percent deviation } \\
\hline 0 & 250.00 & 246.89 & 1.24 \\
0.5 & 226.89 & 224.90 & 1.88 \\
1.0 & 204.16 & 203.17 & 0.49 \\
1.5 & 181.87 & 181.75 & 0.07 \\
2.0 & 160.10 & 160.69 & -0.37 \\
2.5 & 138.95 & 140.08 & -0.82 \\
3.0 & 118.55 & 120.02 & -1.24 \\
3.5 & 99.045 & 100.64 & -1.61 \\
4.0 & 80.635 & 82.118 & -1.84 \\
4.5 & 63.563 & 64.700 & -1.79 \\
5.0 & 48.118 & 48.713 & -1.24 \\
5.5 & 34.622 & 34.580 & 0.12 \\
6.0 & 23.384 & 22.791 & 2.54 \\
6.5 & 14.612 & 13.761 & 5.82 \\
7.0 & 8.3121 & 7.5892 & 8.70 \\
7.5 & 4.2389 & 3.8719 & 8.66 \\
8.0 & 1.9269 & 1.8707 & 2.92 \\
8.5 & 0.7943 & 0.8755 & -10.21 \\
9.0 & 0.3077 & 0.4030 & -30.94 \\
9.5 & 0.1156 & 0.1840 & -59.08 \\
10.0 & 0.0429 & 0.0837 & -139.87 \\
10.5 & 0.0158 & 0.0380 & -194.83 \\
11.0 & 0.0058 & 0.0172 & -266.67 \\
11.5 & 0.0021 & 0.0078 & \\
\hline
\end{tabular}

${ }^{a}$ Data for the dose-dependent example are discussed in the text. 
Table V. Pooled Constants Obtained by Computer Fitting of Equation 3 and by Means of Equations 8 and $9^{a}$

\begin{tabular}{|c|c|c|c|c|c|}
\hline \multicolumn{3}{|c|}{ Computer-fitted values } & \multicolumn{3}{|c|}{ Calculated with equations 8 and 9} \\
\hline$A_{0}$ & $V_{p}$ & $K_{p}$ & $A_{0}$ & $V_{p}$ & $K_{p}$ \\
\hline- & - & - & $x$ & 55 & 27.5 \\
\hline- & - & - & 500 & 53.1 & 26.5 \\
\hline 246.9 & 49.9 & 31.5 & 250 & 51.4 & 25.7 \\
\hline- & - & - & 100 & 47.1 & 23.5 \\
\hline- & - & - & 50 & 41.9 & 21.0 \\
\hline- & - & - & 10 & 28 & 14 \\
\hline 5. & 20.7 & 10.3 & 5 & 23.9 & 11.9 \\
\hline
\end{tabular}

${ }^{a}$ Data were generated by numerical integration of equation 12 using the values of constants given in the text (see dose-dependent example in text).

In pharmacokinetic studies, blood samples are sometimes taken for short fixed intervals of time, irrespective of dose. Each set of dose-dependent data collected in such a manner could appear to be well described by a single Michaelis-Menten equation (each having a pseudolinear LineweaverBurk plot). However, the enzyme constants obtained from these plots or from computer fitting would change dramatically with change in dose. This is further confirmed by use of equations 8 and 9 and is illustrated in Table V. The following trends were evident: First, both $V_{p}$ and $K_{p}$ increased markedly as the dose was increased. Second, the amount of change in $V_{p}$ and $K_{p}$ was dependent on the dose range. The changes in $V_{p}$ and $K_{p}$ were large when $A_{0}$ changed from 5 to 100 , but the change in the constants was relatively small when $A_{0}$ increased from 100 to $\infty$.

\section{General Comments}

As indicated earlier, data generated from parallel Michaelis-Menten equations can sometimes be accurately described at all doses by a single Michaelis-Menten equation. Such "dose-independent" systems can be successfully simplified by using a pooling procedure. Lineweaver-Burk plots constructed from this type of data are pseudolinear. Therefore, linearity of these plots, alone, is not a good criterion of enzyme purity. Moreover, the constants obtained from such a pseudolinear plot, as indicated by equations 8 and 9 , are generally not characteristic of any one enzyme. The values of these constants are a function of all of the microscopic constants for the enzymes.

Dose-dependent systems are characterized by extremely nonlinear Lineweaver-Burk plots and kinetic "constants" which are sensitive to 
large changes in dose. The use of gross enzyme pooling for the simplification of such systems is inappropriate.

A thorough understanding of the pooling concept enables an investigator to determine the suitability of gross enzyme pooling in pharmacokinetic modeling. In order to determine in a specific instance whether pooling may be used to simplify a pharmacokinetic model, studies should be conducted at the extremes of the dose range of interest. If the estimated pooled parameters, obtained by fitting data derived from such a high and a low dose, are not appreciably different and the data are well described by the pooled model, then the system may be considered to be dose independent within the dose range studied. If the sets of data are well described and the constants obtained from the lower-dose data are significantly less than those obtained from the higher-dose data, or the sets of data are not well described by a single Michaelis-Menten equation, the gross pooling of enzymes is inappropriate. The system would have to be further elucidated.

\section{ACKNOWLEDGMENT}

The authors wish to thank Dr. Carl M. Metzler of The Upjohn Company for use of the program NONLIN and his assistance in programming for specific models.

\section{REFERENCES}

1. F. Lundquist and H. Wolthers. The kinetics of alcohol elimination in man. Acta Pharmacol. Toxicol. 14: 265-289 (1958).

2. G. Levy. Pharmacokinetics of salicylate elimination in man. J. Pharm. Sci. 54: 959-967 (1965).

3. G. Levy, T. Tsuchiya, and L. P. Amsel. Limited capacity for salicyl phenolic glucuronide formation and its effect on the kinetics of salicylate elimination in man. Clin. Pharmacol. Therap. 13: 258-268 (1972).

4. N. Gerber and J. G. Wagner. Explanation of dose dependent decline of diphenylhydantoin plasma levels by fitting to the integrated form of the Michaelis-Menten equation. Res. Commun. Chem. Pathol. Pharmacol. 3: 455-466 (1972).

5. J. G. Wagner and J. A. Patel. Variations in absorption and elimination rates of ethyl alcohol in a single subject. Res. Commun. Chem. Pathol. Pharmacol. 4: 61-76 (1972).

6. J. G. Wagner. Properties of the Michaelis-Menten equation and its integrated form which are useful in pharmacokinetics. J. Pharmacokin. Biopharm. 1: 103-121 (1973).

7. J. L. Neal. Analysis of Michaelis kinetics for two independent saturable membrane transport functions. J. Theoret. Biol. 35: 113-118 (1972).

8. G. Spears, J. G. T. Sneyd, and E. G. Loten. A method for deriving kinetic constants for two enzymes acting on the same substrate. Biochem. J. 125:1149-1151 (1971).

9. R. E. Hillman, I. Albrecht, and L. E. Rosenberg. Identification and analysis of multiple glycine transport systems in isolated mammalian renal tubules. J. Biol. Chem. 243: 5566$5571(1968)$.

10. C. R. Schriver and F. Mohyuddin. Amino acid transport in kidney. J. Biol. Chem. 243: 3207-3213 (1968).

11. R. L. Dedrick and D. D. Forrester. Blood flow limitations in interpreting Michaelis constants for ethanol oxidation in vivo. J. Biochem. Pharmacol. 22: 1133-1140(1973). 